\title{
Existence loci of bound states in the continuum in the parameter space of anisotropic planar structures
}

Samyobrata Mukherjee ${ }^{1}$, Jordi Gomis-Bresco ${ }^{1}$, Pilar Pujol-Closa ${ }^{1}$, David Artigas ${ }^{1,2}$, Lluis Torner ${ }^{1,2}$

${ }^{1}$ ICFO-Institut de Ciencies Fotoniques, The Barcelona Institute of Science and Technology, Av. Carl Friedrich Gauss, 3, 08860 Castelldefels (Barcelona), Spain.

${ }^{2}$ Department of Signal Theory and Communications, Universitat Politècnica de Catalunya, Jordi Girona 1-3, 08034 Barcelona, Spain.

Bound states in the continuum (BICs), first predicted in the field of quantum physics [1], are localized radiationless states existing in the part of the spectrum that corresponds to radiative modes. BICs are a general wave phenomenon and have recently been found in photonic systems [2]. They are known to exist in photonic structures in almost-pure transverse-electric (TE) or transverse-magnetic (TM) states, at fixed frequencies and propagation directions [3]. More recently, we have shown the existence of fully hybrid BICs alongside pure TE/TM BICs in planar structures containing uniaxial materials in both symmetric and asymmetric geometries [4]. BICs occur when we decouple the radiation mode from the continuum by suppressing the radiation channel. This can happen in anisotropic planar structures due to polarization separation or destructive interference. The fact that interferometric BICs change propagation direction with frequency suggests that the structure gives itself to potential applications as spatial and spectral filters.

Exploration of the parameter space of the planar structure (Fig 1a) reveals that BICs existence is directly linked to the breaking of the anisotropy symmetry defined by the relative orientation of the optic axes (OAs) of the film and the substrate [5]. Distinct regimes can be identified: (a) anisotropy-symmetric with both OAs in the interface plane and aligned, (b) broken azimuthal symmetry with both OAs in the interface plane but de-aligned and (c) broken polar symmetry with one or both OAs out of the interface plane. We find that the different symmetry breaking not only impacts on the existence loci of BICs, but on their very nature (Fig 1b-d).
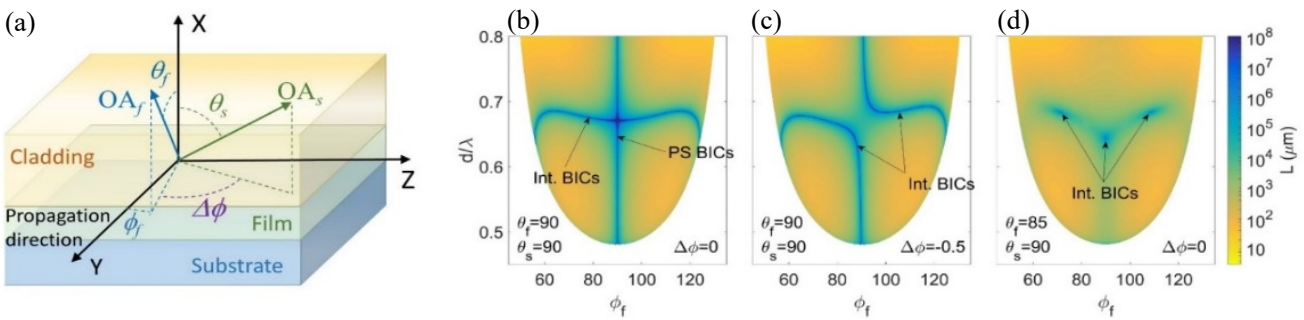

Fig 1. (a) A generic layered waveguide system comprising isotropic cladding, positive birefringent film and negative birefringent substrate. Light propagates along the $\mathrm{Y}$ direction. The green and the blue arrows indicate the substrate and film optical axis (OA), respectively. Structure parameters are as follows: $\left\{\mathrm{n}_{\mathrm{of}}=1.5, \mathrm{n}_{\mathrm{ef}}=1.75, \mathrm{n}_{\mathrm{os}}=2, \mathrm{n}_{\mathrm{es}}=1.25, \mathrm{n}_{\mathrm{c}}=1\right\}$. (b) Existence loci of BICs in an anisotropy symmetric structure. The colored area shows the decay length of the leaky modes in the $\phi_{f}-d / \lambda$ plane, where BICs appear as blue lines. (c) Same as (b) but with azimuthal symmetry broken. (d) Same as (b) but with polar symmetry broken.
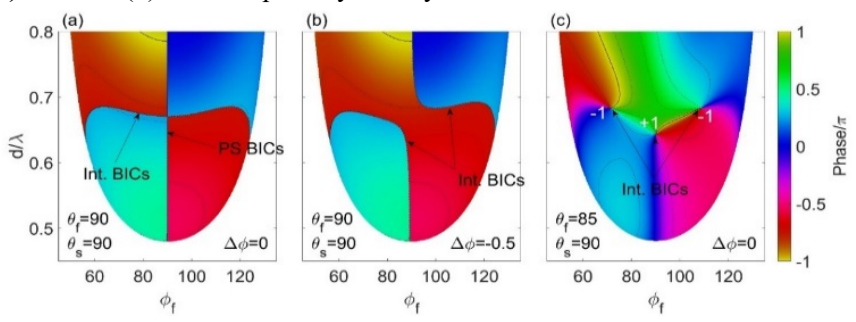

Fig 2. Transformation from phase dislocation (a-b) to screw phase dislocations (c). The representation corresponds to Fig. 2. The color stands for the phase difference of the ordinary radiative channel with respect to the phase of the extraordinary confined wave in the substrate. A winding number of \pm 1 is assigned to the BICs in (c) so that a pair of BIC with opposite sign can be annihilated by parameter tuning.

The phase of the amplitude of the radiation channel surrounding a BIC shows a discontinuity in the anisotropysymmetric and azimuthal symmetry broken structures (Fig 2a, b) whereas a screw phase dislocation is shown in the polar symmetry broken structures (Fig 2c), making BICs very robust under the variation of parameters.

In summary, we reveal that the existence of BICs is strongly affected by the relative orientation of the OAs. We also show that the transition in the existence loci of BICs corresponds to a transition in the phase signature of BICs. The extension to two-dimensional anisotropic structures and the existence of BICs under variation of other system parameters will also be discussed.

\section{References}

[1] J. von Neumann, and E. P. Wigner, “Über merkwürdige diskrete Eigenwerte,” Phys. Z. 30, 465-467 (1929).

[2] C. W. Hsu, et al., "Bound states in the continuum," Nat. Rev. Mater. 1, 16048 (2016).

[3] C. W. Hsu, et al., "Observation of trapped light within the radiation continuum," Nature 499, 188-191 (2013).

[4] J. Gomis-Bresco, D. Artigas and L. Torner, “Anisotropy-Induced Photonic Bound States in the Continuum,” Nat. Photon. 11, $232-236$

(2017).

[5] S. Mukherjee, J. Gomis-Bresco, P. Pujol, D. Artigas and L. Torner, "Topological properties of bound states in the continuum in geometries with broken anisotropy symmetry", Phys. Rev. A 98, 6, 063826 (2018). 